\title{
FMLRC: Hybrid long read error correction using an FM-index
}

\author{
Jeremy R. Wang ${ }^{1 *}+\left(\mathbb{D}\right.$, James Holt ${ }^{2 \dagger}$, Leonard McMillan ${ }^{2}$ and Corbin D. Jones ${ }^{3}$
}

\begin{abstract}
Background: Long read sequencing is changing the landscape of genomic research, especially de novo assembly. Despite the high error rate inherent to long read technologies, increased read lengths dramatically improve the continuity and accuracy of genome assemblies. However, the cost and throughput of these technologies limits their application to complex genomes. One solution is to decrease the cost and time to assemble novel genomes by leveraging "hybrid" assemblies that use long reads for scaffolding and short reads for accuracy.

Results: We describe a novel method leveraging a multi-string Burrows-Wheeler Transform with auxiliary FM-index to correct errors in long read sequences using a set of complementary short reads. We demonstrate that our method efficiently produces significantly more high quality corrected sequence than existing hybrid error-correction methods. We also show that our method produces more contiguous assemblies, in many cases, than existing state-of-the-art hybrid and long-read only de novo assembly methods.

Conclusion: Our method accurately corrects long read sequence data using complementary short reads. We demonstrate higher total throughput of corrected long reads and a corresponding increase in contiguity of the resulting de novo assemblies. Improved throughput and computational efficiency than existing methods will help better economically utilize emerging long read sequencing technologies.
\end{abstract}

Keywords: de novo assembly, Hybrid error correction, Long read, Pacbio, BWT, FM-Index

\section{Background}

De novo genome assembly has benefitted dramatically from the introduction of so-called "long" read sequencing technologies. These technologies, such as SMRT sequencing by Pacific Biosciences (Pacbio) and nanopore sequencing platforms by Oxford Nanopore Technologies, produce reads typically $10 \mathrm{~s}$ of kilobases instead of hundreds of bases. These reads can span repetitive or low-complexity regions of the genome previously unresolvable using only "short"-read next-generation sequencing. Unfortunately, the relatively high error rate of these long-read technologies introduces new informatics and analysis challenges. Effective and efficient methods are necessary to correct these errors in order to realize the potential of these long reads for whole genome assembly [1-4].

\footnotetext{
*Correspondence: jeremy_wang@med.unc.edu

${ }^{\dagger}$ Equal contributors

'Department of Genetics, University of North Carolina at Chapel Hill, CB 3280,

3144 Genome Sciences Building, 250 Bell Tower Dr, Chapel Hill, 27599, NC, USA

Full list of author information is available at the end of the article
}

As the size of long read datasets and genomes undergoing de novo assembly increases, the performance of hybrid long read correction and assembly methods becomes increasingly important. For genomes of more complex eukaryotes and mammals, the computational resources required for effective de novo assembly are staggering and difficult to coordinate. This is driven largely by the pairwise overlap step required by all modern long read assemblers. The time required to overlap these long reads with one another increases quadratically relative to the number of reads. While novel methods such as MHAP [5] and Minimap [6] aim to improve this, in practice, the computational time and memory required are often prohibitively expensive.

Pre-assembly correction dramatically simplifies the subsequent overlap and layout of long reads for assembly by reducing the variance that must be accounted for in the overlapping step. In particular, long reads having undergone error correction are likely to share much longer identical stretches that can be used to efficiently find 
confidently overlapping reads. Fundamentally, the longer and more accurate these corrections are, the more quickly and accurately the long reads can be assembled.

Long read correction algorithms can be broadly classified as either self-correction or hybrid correction algorithms. Self-correction algorithms correct long reads using only other long read sequences. Self-correcting algorithms, including Sprai [7], LoRMA [8], HGAP [1], and $\mathrm{PBCR}$ [3] align the long reads to each other and generate a consensus sequence. In order to generate an accurate consensus, these methods require relatively high coverage of long read sequence to overcome the high error rate. Unfortunately, the relatively high cost per accurate nucleotide for long-read sequencing technologies means that deep sequencing using only long reads is expensive.

In contrast, hybrid correction algorithms use shortread sequencing of the same sample to complement and correct the long reads. Short-read sequencing has fewer sequencing errors, costs less per base sequenced, and thus the cost per accurate nucleotide is much lower. Many hybrid error correction methods act similar to scaffolders in that they require the assembly of complementary short read data first, then alignment between long reads and short-read unitigs or contigs. These approaches, while reasonably effective, suffer from two classes of problems. First, they incur the same type of disadvantages a shortread only assemblies in that low-complexity and repetitive elements larger than the size of the short reads cannot be reliably resolved. When short reads are preassembled, this bias can "correct" long read with incorrect sequence, confounding assembly. Second, short read assembly followed by pairwise alignment/overlap of long reads with short-read contigs is often significantly slower than direct long-read error correction.

Hybrid correction algorithms include LoRDEC [4], ECTools [9], Jabba [10], CoLoRMap [11], and Nanocorr [12]. Other methods, including Cerulean [13], DBG2OLC [14], and hybridSPAdes [15] perform hybrid assembly of long- and short-read data but do not explicitly correct errors in the long-read sequences. These hybrid methods are often able to construct more accurate and contiguous assemblies than exclusively long-read assembly methods at substantially lower cost. ECTools [9] and Nanocorr [12] are based on the same underlying methodology, but designed for Pacbio and nanopore sequences, respectively. They perform a full alignment between short and long reads, but are currently deprecated and take prohibitively long to run for anything larger than microbial genomes, so they were not considered further.

For error correction or assembly methods to be useful for large, complex genomes that are biomedically or economically important, the key challenge is performing as accurate an assembly as possible, as quickly as possible, and using as few computational resources as possible.
Current methods often require prohibitively large computational resources. Given that finding the appropriate parameters for an assembly is often an iterative process, these high computational costs are a barrier.

\section{Methods}

We introduce a new hybrid method for correcting errors in long-read sequences called FM-index Long Read Corrector (FMLRC). The main contribution of our method is the application of an FM-index built from a multi-string Burrows-Wheeler Transform (BWT) [16] of the short-read sequencing datasets. The FM-index enables arbitrary length $k$-mer searches through the dataset, allowing for FMLRC to retrieve $k$-mer frequencies from the short-read dataset in $O(k)$ steps. Unlike other data structures, the length of $k$ is not fixed during construction of the FM-index but is instead selected at run-time. As a result, FMLRC uses the FM-index to implicitly represent all de Bruijn graphs [17] of the shortread sequencing dataset. These de Bruijn graphs are then used to correct regions in the long reads that are not supported by the short-read sequencing dataset.

Two secondary contributions arise as a result of the first. FMLRC uses the single FM-index data structure to perform two correction passes over each read: first with a short $k$-mer and second with a longer $K$-mer. Secondly, the specific parameters of the correction algorithm are dynamically adjusted to match the $k$-mer frequencies for a given read at run-time. FMLRC takes as input a BWT of the short-read sequencing dataset. It constructs a single FM-index in memory that is shared across all processes. Each process individually corrects one read at a time by applying common de Bruijn graph correction methods (namely seed-and-extend or seed-and-bridge) using the shared FM-index. These de Bruijn correction methods require both a $k$-mer size and frequency thresholds to determine whether a $k$-mer is present in the graph. FMLRC dynamically adjusts these thresholds at run-time for each pass over a long read. A single process will correct the read using the implicit short $k$-mer de Bruijn graph and then the implicit long $K$-mer de Bruijn graph before writing the corrected result to disk. An overview of this approach is shown in Fig. 1.

FMLRC is a publicly available $\mathrm{C}++$ program ${ }^{1}$. The implementation requires construction of a BWT of the short-read dataset in the run-length encoded format of the msbwt package ${ }^{2}$.

\section{Advantages of the FM-index}

FMLRC can be classified as a de Bruijn graph-based, hybrid read corrector, meaning it uses $k$-mer frequencies from a short-read sequencing dataset to correct errors in a long-read sequencing dataset. Generally speaking, most de Bruijn graph implementations are static and require 


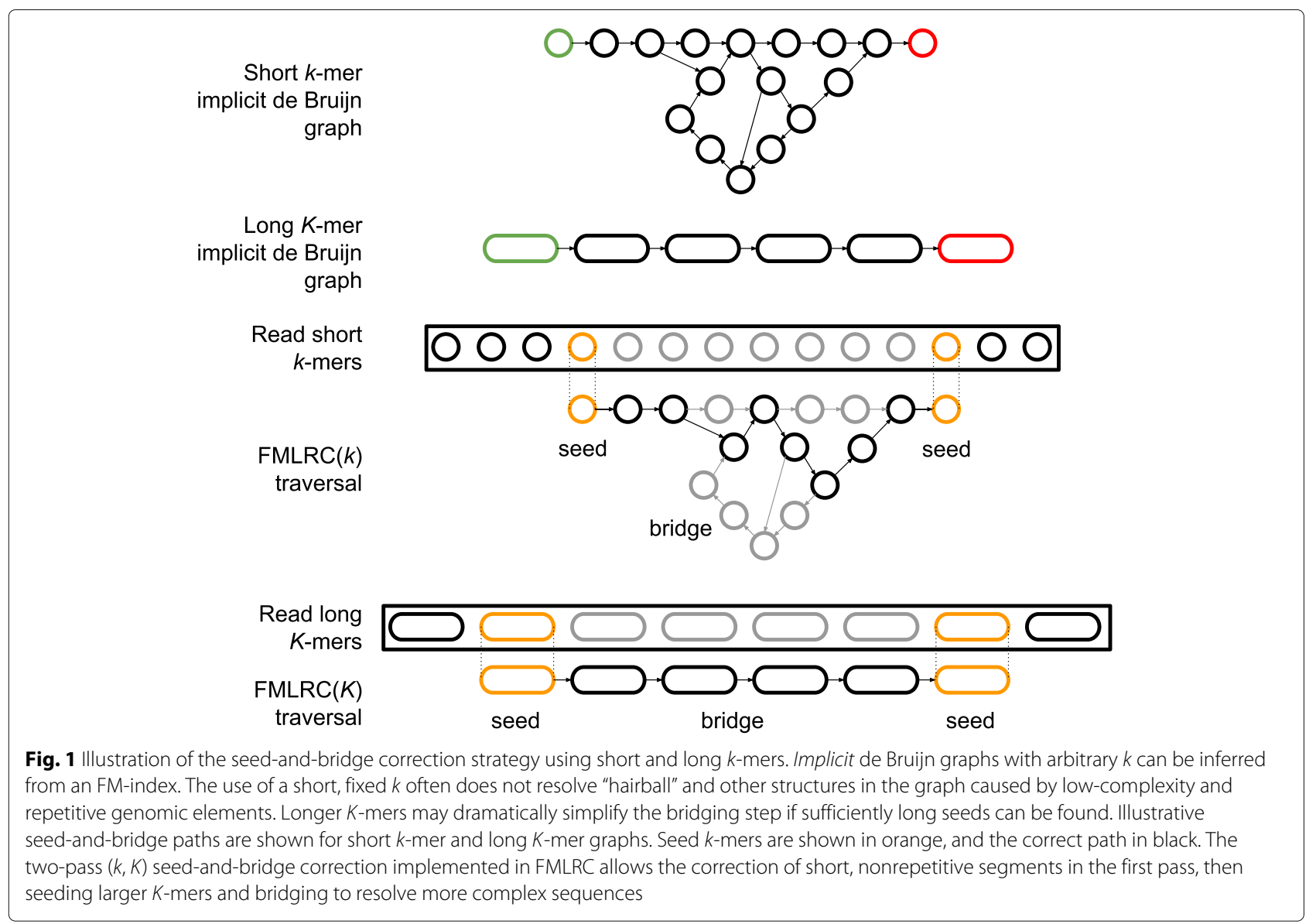

a fixed $k$-mer size and pruning threshold to be defined during the construction of the de Bruijn graph [17].

The main advantage of FMLRC is that it uses an FMindex as the underlying de Bruijn graph implementation. FMLRC builds an FM-index [18] from a BWT [19] of a short-read sequencing dataset to correct a long-read sequencing dataset. These data structures have been previously used for short-read self-correction in FMRC [20], but it has not been previously applied to long-read error correction.

The FM-index is advantageous because many of the correction parameters are not properties of the data structure itself and can instead be defined and/or dynamically adjusted at run-time. First, FM-index queries are not fixed to a single $k$-mer size, allowing FMLRC to construct one FM-index and use it for all $k$-mer queries. Secondly, the FM-index is built from a BWT that is a lossless encoding of the original reads, meaning that no $k$-mers are "pruned" as they commonly are in a de Bruijn graph. This pruning is usually accomplished by removing all $k$-mers with a frequency less than a fixed threshold. Instead, FMLRC dynamically calculates thresholds for each long read and decides whether a $k$-mer is "pruned" at runtime. The combination of these two properties means the
FM-index implicitly represents all de Bruijn graphs for the short-read sequencing dataset.

FMLRC creates an in-memory FM-index by scanning the BWT from disk. There are many different implementations of in-memory FM-indices that have varying trade-offs between memory usage and CPU time to perform a $k$-mer lookup. FMLRC currently has two FM-index implementations. The default FM-index implementation uses bit arrays and rank operations to enable fast $k$-mer lookups. This primary implementation sacrifices memory usage to increase computational performance. The second FM-index implementation is a traditional sampled FMindex that allows users to set the sampling rate, leading to longer computations with a smaller memory footprint. The two FM-index implementations produce identical corrected read results, and we present only the results from the primary implementation in our performance results.

For our results, we constructed the BWTs using a combination of ropebwt2 [21] and the msbwt package ${ }^{3}$. While this particular format stores only the original read sequences, we must consider both the forward and reverse-complement sequences when performing $k$-mer queries. Every time we refer to a $k$-mer query, FMLRC 
is actually querying both the forward and reversecomplement sequences and adding their frequencies together prior to performing any checks.

\section{De Bruijn graph-based correction}

FMLRC accesses implicit, pruned, $k$-mer de Bruijn graphs through the FM-index. While the de Bruijn graph-based correction of FMLRC is similar to that of LoRDEC [4], we briefly describe it here for completion and for reference in the following sections. Given a long read and a de Bruijn graph, the first step is to classify all $k$-mers in the long read as either weak or solid. In general, solid $k$-mers are supported by the de Bruijn graph and weak $k$-mers are not. For each $k$-mer in the long read, its $k$-mer frequency is retrieved from the de Bruijn graph. If that frequency is below a threshold, $t$, it is consider weak and otherwise it is considered solid. Weak regions are consecutive weak $k$-mers in the long read. Solid regions are consecutive solid $k$-mers in the long read.

Weak regions can be flanked by zero, one, or two solid regions. If a weak region has no flanking solid regions, the entire read is one large weak region with no solid $k$-mers to initialize a traversal of the de Bruijn graph. As a result, these reads are not changed because there are no start points for a de Bruijn graph traversal.

If a weak region has one flanking solid region, then it is either a head or tail weak region in the read. In either case, the solid $k$-mer closest to the weak region is used as a "seed" $k$-mer for traversing the de Bruijn graph. FMLRC performs a depth-first traversal of the de Bruijn graph from this seed using an expected path length based on the size of the weak region and returns any found paths (seed-and-extend). If a weak region has two flanking solid regions, FMLRC uses the two closest $k$-mers from each solid region as "seed" and "target" $k$-mers (seedand-bridge). FMLRC then performs a depth-first traversal from the seed $k$-mer and returns any paths that connect to the target $k$-mer. If no path is found, FMLRC attempts to extend backwards from the target to the seed $k$-mer, which may resolve additional bridges that have excessive branching close to the seed $k$-mer. If any paths are returned from a de Bruijn graph traversal, the paths are compared to the original weak region and the one with the smallest edit distance is chosen to replace it. If no paths are returned, then no change is made to the long read at that region. In all de Bruijn graph traversals, we prevent exponential traversal time by enforcing a branching limit, $L$. Typically, the parameters $t$ and $L$ are either constant values in a program or user-defined static values.

\section{Differences in the short and long passes}

One of the key differences in FMLRC compared to other approaches is that it accesses two different de Bruijn graphs though the FM-index and dynamically adjusts the parameters of the correction algorithm to adjust for differences in the graphs. FMLRC performs two passes: the first with a short $k$-mer size and the second with a longer $K$-mer size. For FMLRC, the two passes are programmatically identical with the value of $k$ or $K$ passed as a parameter. For brevity, we describe the differences in each pass using parameter $k$ noting that replacing $k$ with $K$ describes the second pass of our method. Additionally, we describe any dynamic variables as functions of $k$, the implicit $k$-mer de Bruijn graph, and other user-defined constants.

In general, the short $k$-mer pass does the majority of the correction for FMLRC, whereas the longer $K$-mer pass tends to correct repetitive, low-complexity regions within the long read. To provide some intuition behind why the long pass improves the results, we focus on the differences in de Bruijn graphs representing the same data but with two different $k$-mer lengths. In general, two distinct paths will be merged in a $k$-mer de Bruijn graph if they share a pattern that is at least $k$ long. This is because the nodes along that shared region will be identical. At the ends of the shared region, there will be two paths emerging representing the differences at the edge of the shared regions.

When the same sequences are viewed through a longer $K$-mer de Bruijn graph, the number of merged, ambiguous paths strictly decreases because an increasing amount of similarity is required for the paths to become merged in the graph. This effect is illustrated in Fig. 1. In practice, short $k$-mers are often long enough to uniquely identify most areas of the genome. However, genomic characteristics such as low-complexity sequence, gene families, or repeat regions are difficult to traverse using short $k$-mers. Thus, our method uses the larger $K$-mer to bridge weak regions composed of repeated or low-complexity sequences that are computationally expensive to fully traverse using a small $k$-mer.

In addition to changing the value of $k$ in the two passes, other parameters are adjusted as well to match the different $k$-mer sizes. First, the threshold, $t$, determining whether a $k$-mer is weak or solid is dynamically adjusted for each long read. FMLRC uses a dynamic threshold based on the $k$-mer frequencies in the long read. First, there is an absolute minimum, user-defined $k$-mer frequency, $T$, that is required for any $k$-mer to be consider solid. Second, the frequency of any $k$-mer greater than this absolute minimum is added to a list and used to calculate a median solid frequency, $m$, for the long read. A second user-defined value, $F$, is the fraction of this median that is required for a $k$-mer to be considered solid. Thus, the final threshold distinguishing solid and weak $k$-mers in a given long read is defined as $t=\max (T, F * m)$. In summary, with each pass over a long read, FMLRC dynamically calculates a threshold for determining weak 
or solid $k$-mers based on an absolute minimum and the surrounding $k$-mer frequencies from the long read.

For low-coverage short-read datasets, it is often the case that $t=T$ because $F * m<T$. For high-coverage short-read datasets, this dynamic threshold alleviates the need to select a fixed threshold beforehand, and it instead uses counts from the implicit de Bruijn graph to derive an expected count for $k$-mers in the read. Additionally, this approach enables FMLRC to adjust the threshold for different sizes of $k$ automatically at run-time.

Finally, the branch limit, $L$, is scaled with each pass to allow for less branching when $k$ is small and more branching when $K$ is large. As described earlier, a small $k$-mer de Bruijn graph will have more branches and may require more computation to do a full depth-first traversal in repetitive regions. To avoid this, we restrict the short $k$ mer traversals to primarily fixes the "easy" errors caused by sequencing. As a result, the "harder" traversals caused by larger repetitive elements are addressed more accurately by the long $K$-mer pass. The branch limit factor, $B$, is a user defined parameter such that the maximum branch limit, $L=B * k$.

\section{FMLRC parameter selection}

FMLRC allows for five main parameters to be defined by the user: $T, F, B, k$, and $K . T$ is the absolute minimum frequency required for a $k$-mer to be considered solid in the de Bruijn graph. $F$ is the fraction of the median counts required for a $k$-mer to be considered solid in the de Bruijn graph. $B$ is the branch limit factor that limits the amount of computation of a de Bruijn graph traversal. In all test cases, we used the FMLRC default parameters: $T=5$, $F=0.10$, and $B=4$.

The last two parameters are the choice of $k$ and $K$ for the short and long correction passes. To gain some insight into what values of $k$ and $K$ are best, we ran multiple tests using the $E$. coli K12 MG1655 and S. cerevisiae W303 datasets. We allowed $k=[17,19,21,23,25]$ and $K=[-, 49,59,69,79,89]$, leading to a total of 30 test cases for each dataset. The test cases with $K=-$ indicate that no second $K$-mer pass was performed (it is only using a one-pass, short $k$-mer for correction). For each test case, we ran FMLRC, aligned the corrected reads to the reference genome, and then gathered statistics on the resulting alignment. We counted the the total number of bases that matched the reference genome and the "gain" (see "Correction accuracy" section). The results of this experiment are shown in Table 1.

We see that as $k$ and $K$ increase, gain generally increases but the total number of matching bases decreases, indicating a tradeoff between sensitivity and specificity. In all of our tests, performing a second pass with the long $K$ mer always improved all three statistics. In general, the gain begins plateauing around $k=21$ and $K=59$ and matching bases decreases in the E. coli dataset, so we chose these as the default values for $k$ and $K$. While it is clear that the "best" $k$ and $K$ is likely data-dependent because differences in coverage, sequencing quality, and sequencing content will impact the ability of FMLRC to find solid $k$-mers and perform corrections, these defaults perform close to optimal across all of our evaluated datasets.

\section{Results}

We evaluated the accuracy of our method using complementary long- and short-read datasets for three species: E. coli K12, S. cerevisiae W303, and A. thaliana Ler-0 (see "Availability of data and material" section). We compared the relative correction accuracy and computational performance of our method to several existing hybrid and long-read-only correction methods. We also assessed the effectiveness of our corrected reads for de novo assembly using a non-correcting assembler, Miniasm [6], and compared these data to several other state-of-the-art hybrid and long-read-only de novo assembly methods.

\section{Correction accuracy}

To evaluate FMLRC, we used the approach used by the Error Correction Evaluation Toolkit (ECET) [23] to calculate error correction sensitivity, specificity, and "gain" relative to a known reference genome (Sensitivity = $T P /(T P+F N)$, Specificity $=T N /(T N+F P)$, and gain $=$ $(T P-F P) /(T P+F N)$ where TP, TN, FP, and FN are true positives, true negatives, false positive, and false negative, respectively). We modified the published pipeline to work efficiently with long reads, but the statistics are computed in an similar manner. In particular, we aligned the original and corrected FASTA files to the corresponding reference genome for each organism using BLASR [22]. Using the original ECET implementation, which was designed for short-read sequences, specific loci in long reads could not be evaluated before and after error correction due to the high incidence of short insertions and deletions. Instead, we consider loci relative to the reference sequence to which each read aligned. A nucleotide is considered "correct" if it aligns properly to a single nucleotide in the read sequence. Loci in the reference sequence with mismatched or delected nucleotides in the read sequence are considered incorrect. Our evaluation code is available at https://github.com/txje/lrc_eval, including the computation of error correction statistics directly from BLASR's $-m 5$ format alignments.

In addition to these statistics, we report the total aligned reads and properly aligned nucleotides. Again unlike short-read error correction, where every read is expected to align in full both before and after error correction, the number and span of long-read alignments may fluctuate and impacts the utility of a sequence dataset for 
Table 1 Choosing $k$ and $K$

\begin{tabular}{|c|c|c|c|c|c|c|}
\hline \multirow[b]{2}{*}{ k } & \multicolumn{6}{|l|}{ K } \\
\hline & - & 49 & 59 & 69 & 79 & 89 \\
\hline \multicolumn{7}{|c|}{ E.coli - Matching Bases } \\
\hline 17 & 404736174 & 405150086 & 404809579 & 404608992 & 404361044 & 403888297 \\
\hline 19 & 403117580 & 404580392 & 404571352 & 404418084 & 404297325 & 403826761 \\
\hline 21 & 403002237 & 404365615 & 404367089 & 404255830 & 404131272 & 403841312 \\
\hline 23 & 403516577 & 404381062 & 404378242 & 404240202 & 404107504 & 404041491 \\
\hline 25 & 403819785 & 404461301 & 404480970 & 404453527 & 404363292 & 404236385 \\
\hline \multicolumn{7}{|c|}{ E. coli - Gain } \\
\hline 17 & 0.1011 & 0.388 & 0.4709 & 0.5258 & 0.5468 & 0.521 \\
\hline 19 & 0.3823 & 0.5887 & 0.612 & 0.6245 & 0.6279 & 0.6172 \\
\hline 21 & 0.4879 & 0.634 & 0.6429 & 0.6459 & 0.6442 & 0.6345 \\
\hline 23 & 0.5137 & 0.641 & 0.6474 & 0.6487 & 0.6457 & 0.6361 \\
\hline 25 & 0.523 & 0.6396 & 0.6453 & 0.6461 & 0.6422 & 0.6318 \\
\hline
\end{tabular}

S. cerevisiae - Matching Bases

$\begin{array}{lllllll}17 & 1250679980 & \mathbf{1 2 5 3 5 9 0 9 9 0} & 1252340540 & 1251288299 & 1250445441 & 1249925285 \\ 19 & 1250052124 & 1252517259 & 1252462544 & 1252139063 & 1251853858 & 1251785285 \\ 21 & 1248322270 & 1251887685 & 1251963458 & 1251672602 & 1251758116 & 1251744201 \\ 23 & 1248801294 & 1252245368 & 1252387319 & 1252408890 & 1252545735 & 1252558864 \\ 25 & 1249574404 & 1252269051 & 1252478532 & 1252557840 & 1252778626 & 1252739127\end{array}$

S. cerevisiae - Gain

\begin{tabular}{lllllll}
17 & 0.0264 & 0.224 & 0.3159 & 0.3946 & 0.452 & 0.4871 \\
19 & 0.1172 & 0.3903 & 0.443 & 0.4822 & 0.5096 & 0.5273 \\
21 & 0.2527 & 0.4938 & 0.5129 & 0.527 & 0.5367 & 0.5434 \\
23 & 0.3319 & 0.5153 & 0.5251 & 0.5332 & 0.5388 & $\mathbf{0 . 5 4 3 5}$ \\
25 & 0.3728 & 0.5155 & 0.5226 & 0.5287 & 0.5334 & 0.5372 \\
\hline
\end{tabular}

This table shows the result of running FMLRC using many different values for $k$ and $K$ for an $E$. coli and S. cerevisiae datasets

The test cases with $K=-$ indicate that no second pass of correction using the long $K$-mer was performed, so those test cases use a single pass short $k$-mer only. After correcting the reads, we aligned the results using BLASR [22] and gathered statistics on the alignments. Matching bases indicates the number of matching bases across all mappings. Gain is defined as (TP - FP)/(TP + FN) (see "Correction accuracy" section). For each statistic, the best result is bolded in the above table. To summarize, increasing values for $k$ and $K$ tend to increase the gain but decrease the total matching bases - a tradeoff between sensitivity and specificity. Additionally, all tested values of $K$ for a long $K$-mer pass improves the results over a single $k$-mer pass

downstream analysis. For example, error correction methods that agressively filter out low-quality sequences, such as Jabba [10], may report very high sensitivity and specificity, but do so by reporting and aligning only a subset of the input sequences.

In addition to evaluating FMLRC, we also evaluated the following hybrid correction methods using the same ECET pipeline: LoRDEC [4], Jabba [10], and CoLoRMap [11]. For completeness, we also included comparison to long-read-only methods: Canu [5], LoRMA [8], and Sprai
[7]. For all tests, we ran LoRMA v0.4, LoRDEC v0.6 with options -k 21 - S 5, and Jabba with option -k 75 (as recommended in [10]). FMLRC was run with default parameters (-k 21 -K 59) for E. coli, S. cerevisiae, and A. thaliana. All other methods' parameters were left at their defaults.

Table 2 shows accuracy metrics and resource usage for all compared methods. For A. thaliana and S. cerevisiae, FMLRC has the highest total corrected loci (true positives) and competitive gain and sensitivity. For 
Table 2 After aligning the corrected reads to a reference genome, sensitivity, specificity, and gain were computed

\begin{tabular}{|c|c|c|c|c|c|c|c|c|}
\hline Method & Reads aligned & $\mathrm{TP}$ & FN & Sens. & Spec. & Gain & $\mathrm{CPU}(\mathrm{s})$ & Mem (GB) \\
\hline \multicolumn{9}{|l|}{ E. coli K12 } \\
\hline Canu & 58982 & 657793 & 1571579 & 0.2951 & 0.9987 & 0.1482 & 17421 & 2.99 \\
\hline CoLoRMap & 81485 & 5538038 & 35474190 & 0.1350 & 0.9998 & 0.1332 & 137777 & 23.06 \\
\hline FMLRC & 81851 & 13562639 & 15069242 & 0.4737 & 0.9996 & 0.4689 & 11015 & 4.60 \\
\hline Jabba & 75620 & 15553372 & 111609 & 0.9929 & 0.9999 & 0.9920 & 22922 & 63.87 \\
\hline LORDEC & 81138 & 3278911 & 36691424 & 0.0820 & 0.9998 & 0.0808 & 61305 & 2.08 \\
\hline LORMA & 81051 & 1135657 & 144161 & 0.8874 & 0.9999 & 0.8669 & 54240 & 45.72 \\
\hline Sprai & 75532 & 463636 & 293039 & 0.6127 & 0.9999 & 0.5783 & 44302 & 33.53 \\
\hline \multicolumn{9}{|c|}{ S. cerevisiae W303 } \\
\hline Canu & 142765 & 1562542 & 5441239 & 0.2231 & 0.9992 & 0.1401 & 108175 & 3.33 \\
\hline CoLoRMap & 210423 & 18901871 & 79065115 & 0.1929 & 0.9992 & 0.1857 & 2815200 & 45.49 \\
\hline FMLRC & 211270 & 31849332 & 49204291 & 0.3929 & 0.9991 & 0.3829 & 68519 & 17.77 \\
\hline Jabba & 223385 & 29893606 & 42670 & 0.9986 & 0.9999 & 0.9967 & 187968 & 367.92 \\
\hline LoRDEC & 210151 & 8468872 & 96577493 & 0.0806 & 0.9997 & 0.0776 & 212495 & 3.56 \\
\hline LoRMA & 204323 & 3063164 & 221583 & 0.9325 & 0.9999 & 0.9176 & 223358 & 49.52 \\
\hline Sprai & 192670 & 2013269 & 3063751 & 0.3965 & 0.9996 & 0.3288 & 215261 & 49.52 \\
\hline \multicolumn{9}{|c|}{ A. thaliana Ler-0 } \\
\hline Canu & 574065 & 12002535 & 72017120 & 0.1429 & 0.9986 & 0.1030 & 1301971 & 10.92 \\
\hline CoLoRMap & 1075381 & 170235345 & 2056204621 & 0.0765 & 0.9983 & 0.0737 & 6802359 & 106.08 \\
\hline FMLRC & 1447042 & 442064624 & 1164804073 & 0.2751 & 0.993 & 0.2601 & 708910 & 16.26 \\
\hline Jabba & 813495 & 320742341 & 2945173 & 0.9909 & 0.9968 & 0.9724 & 3641309 & 333.44 \\
\hline LoRDEC & 1113617 & 78276025 & 2022520259 & 0.0373 & 0.9979 & 0.0337 & 1111800 & 3.42 \\
\hline LoRMA & 903298 & 2217661 & 2778223 & 0.4439 & 0.9986 & 0.3715 & 17281259 & 70.28 \\
\hline Sprai & 751684 & 18960255 & 30734331 & 0.3815 & 0.9996 & 0.3631 & 5996657 & 8.11 \\
\hline
\end{tabular}

For A. thaliana and S. cerevisiae, FMLRC produced more total true positive (corrected loci) than any other method while maintaining competitive sensitivity and gain. Methods with higher average specificity, notably Jabba, often discard a higher proportion of reads, reporting only those with the highest-confidence corrected sequence. FMLRC also requires significantly less CPU time than other hybrid error correction methods, and comparable memory. LoRDEC and FMLRC CPU time and memory results include construction of the BWT

E. coli, FMLRC corrects fewer loci than Jabba, but more total reads. As discussed above, methods with higher sensitivity and specificity - including LoRMA, Sprai, and Jabba - typically accomplish this by selectively reporting the highest-confidence corrected sequences. This kind of confidence filtering is possible after correction for most methods, but can negatively impact downstream assembly (see "De novo assembly" section).

\section{Performance}

CPU and memory usage for each method are shown in Table 2. Performance tests were run on a homogenous cluster of 120 compute nodes, each with two Intel E2680 $(2.5 \mathrm{GHz})$ processors and $1 \mathrm{~Tb}$ RAM. FMLRC requires less $\mathrm{CPU}$ time (including construction of the general-purpose BWT) than all other hybrid correction methods. On average, FMLRC's memory usage is among the most memory-efficient methods, including Canu and LoRDEC. The remaining hybrid error-correction methods, CoLoRMap, LoRMA, and Jabba, use significantly more memory and, especially in the case of Jabba (>300GB) may prove prohibitive to run without significant computational infrastructure. Jabba, in particular, while producing comparable total true positives to FMLRC, required $2-5 \times$ as much CPU time and $15-20 \times$ as much memory.

\section{De novo assembly}

The ultimate goal of any long read correction algorithm is to provide better data for genomic analysis. We assessed the ability of our method to successfully complete assembly of simple and complex genomes and to compare its performance to other long-read error correction and 
de novo assembly methods. We assessed the methods listed in Table 3 on the E. coli, S. cerevisiae, and A. thaliana datasets listed above. Our method, along with LoRDEC and Sprai, perform only read correction. We used Miniasm (https://github.com/lh3/miniasm r159 and Minimap https://github.com/lh3/minimap r124) to assemble the corrected reads from these methods. We used option $-S w 5$ for Minimap; all other parameters were left at their defaults. The straightforward approach to identity-based overlapping and graph layout used by Miniasm allows us to assess the effect of read correction on de novo assembly.

All assemblies were run on a heterogeneous Linuxbased cluster with more than 9600 cores and $48 \mathrm{~Gb}-1 \mathrm{~Tb}$ RAM per node. All jobs had a hard limit of 16 processes and 7 days wall-clock run time. For larger genomes such as A. thaliana, several methods, including hybridSPAdes and Cerulean, failed after exceeding these limits or exceeding $1 \mathrm{~Tb}$ main memory. Canu is a modern fork of the Celera Assembler and consists of the basic PBcR correction method using the MHAP overlapper followed by assembly with HGAP. So we assess only the Canu pipeline as a whole.

Several of the methods took prohibitively long ( $>1$ week) or failed to assemble the A. thaliana genome. We analyzed completed assemblies using Quast v4.1 [24] with default parameters in Table 4. Percent error indicates the total of mismatched bases, insertions/deletions, and no-calls (Ns). As shown, FMLRC has comparable performance to other methods for E. coli K12. It also outperforms all methods except Canu in terms of N50 for $S$. cerevisiae W303. Although the continuity is often higher

Table 3 Long-read and hybrid correction and assembly methods

\begin{tabular}{|c|c|c|c|c|}
\hline Method & Correction & Assembly & Preassembly & Citation \\
\hline Miniasm & & Long-read & & {$[6]$} \\
\hline Canu & Long-read & Long-read & & {$[5]$} \\
\hline Sprai & Long-read & & & {$[7]$} \\
\hline LoRMA & Long-read & & & {$[8]$} \\
\hline hybridSPAdes & & Hybrid & & {$[15]$} \\
\hline DBG2OLC & & Hybrid & $x$ & {$[14]$} \\
\hline Cerulean & & Hybrid & $x$ & [13] \\
\hline ECTools & Hybrid & & $x$ & {$[9]$} \\
\hline LoRDEC & Hybrid & & & [4] \\
\hline Jabba & Hybrid & & & {$[10]$} \\
\hline CoLoRMap & Hybrid & & & [11] \\
\hline Nanocorr & Hybrid & & & {$[12]$} \\
\hline FMLRC & Hybrid & & & Our method \\
\hline
\end{tabular}

All of the compared methods are shown along with their mode of error correction and assembly, each either long-read only or "hybrid" using complementary short-read data. "Preassembly" indicates whether a hybrid method requires the short read data to be preassembled using a different method for Canu and other long-read consensus methods, these typically rely on high coverage of long reads and degrade in performance as coverage drops. These test datasets contain high $(>100 \times)$ coverage of both long and short reads. Furthermore, post-assembly polishing steps such as Quiver [1] and Nanopolish [25] are typically effective in reducing the assembly error from less than $1 \%$ to less than $0.01 \%$.

\section{Discussion}

Correction of errors in long read sequences using complementary short reads remains a popular method for increasing the utility of long read sequence, particularly since long read sequencing remains prohibitively expensive relative to standard NGS in many cases. While several methods exist for hybrid error correction and assembly $[4,9-15]$, these approaches sometimes limit the utility of corrected sequences for downstream assembly or other applications due to low throughput - they report only segments where very high accuracy can be achieved or clip and trim low confidence sequences. These produce very polished (accuracy in excess of 99\%) sequence, but reduce the total number and size of sequences available for assembly. In practice, a balanced approach is necessary to retain the long-range information while increasing sequence accuracy to aid in pairwise overlapping of reads.

Our proposed method does not perform any clipping or trimming of long read sequences, but corrects errors using high-accuracy short read sequences, enabling more sensitive and specific overlap of reads during de novo assembly. While no method produces obviously better results across all assembly metrics, FMLRC exhibits high accuracy correction while maintaining high assembly contiguity for a range of genome sizes. Practically, our method is also computationally efficient whereas competitive methods such as Jabba take prohibitive computational resources for even moderately sized data sets.

\section{Conclusion}

Flexible "modular" approaches to de novo long read sequence assembly are becoming more popular with the introduction of efficient overlap and layout methods such as DALIGNER (https://github.com/thegenemyers/ DALIGNER), MHAP [5], Minimap [6], and Miniasm [6]. Existing error correction methods including DBG2OLC [14], Cerulean [13], and hybridSPAdes [15] require preassembly of short read sequence and perform a variant of scaffolding using long read sequences. While this approach benefits from the high accuracy of short read sequence, it retains the biases inherent in assembly of short read sequences. In particular, it is often difficult or impossible to properly assemble low-complexity or repetitive sequences using only short reads [26]. 
Table 4 Long-read and hybrid correction assembly statistics

\begin{tabular}{|c|c|c|c|c|c|}
\hline Dataset & Method & \# contigs & N50 & Genome fraction & Error rate \\
\hline E. coli K12 & Canu + Miniasm & 1 & 4631922 & 99.832 & 0.00444 \\
\hline \multirow{11}{*}{$\begin{array}{l}\text { Genome: 5Mb } \\
\text { Pacbio: } 450 \mathrm{Mb} \\
\text { Illumina: 3.4Gb }\end{array}$} & CoLoRMap + Miniasm & 1 & 4723063 & 84.485 & 0.02322 \\
\hline & FMLRC + Miniasm & 1 & 4646838 & 99.757 & 0.00029 \\
\hline & Jabba + Miniasm & 76 & 72751 & 92.923 & 0.00005 \\
\hline & LoRDEC + Miniasm & 1 & 4688727 & 97.504 & 0.00321 \\
\hline & LoRMA + Miniasm & 107 & 64214 & 89.871 & 0.00099 \\
\hline & Sprai + Miniasm & 1 & 4639974 & 99.989 & 0.00092 \\
\hline & Miniasm & 1 & 4783415 & 0.002 & 0.01333 \\
\hline & Canu & 2 & 4656585 & 99.998 & 0.00012 \\
\hline & hybridSPAdes & 2 & 4469733 & 99.967 & 0.0001078 \\
\hline & DBG2OLC & 2 & 4585967 & 98.210 & 0.00225 \\
\hline & Cerulean & 16 & 1258842 & 98.959 & 0.09500 \\
\hline \multirow{2}{*}{ S. cerevisiae W303 } & Canu + Miniasm & 36 & 729798 & 89.739 & 0.00521 \\
\hline & CoLoRMap + Miniasm & 23 & 766539 & 83.464 & 0.00761 \\
\hline \multirow{10}{*}{$\begin{array}{l}\text { Genome: 12Mb } \\
\text { Pacbio: 1.3Gb } \\
\text { Illumina: 18Gb }\end{array}$} & FMLRC + Miniasm & 32 & 771324 & 87.717 & 0.00175 \\
\hline & Jabba + Miniasm & 186 & 62337 & 72.239 & 0.00008 \\
\hline & LoRDEC + Miniasm & 61 & 597849 & 85.563 & 0.00941 \\
\hline & LoRMA + Miniasm & 292 & 49850 & 74.632 & 0.00103 \\
\hline & Sprai + Miniasm & 39 & 561985 & 88.697 & 0.00193 \\
\hline & Miniasm & 29 & 566484 & 0.009 & 0.03738 \\
\hline & Canu & 26 & 777664 & 90.955 & 0.00068 \\
\hline & hybridSPAdes & 229 & 568823 & 87.303 & 0.00899 \\
\hline & DBG2OLC & 32 & 530806 & 0.067 & 0.03407 \\
\hline & Cerulean & 78 & 466556 & & 0.03687 \\
\hline A. thaliana Ler-0 & Canu + Miniasm & 2100 & 74153 & 81.844 & 0.01410 \\
\hline \multirow{9}{*}{$\begin{array}{l}\text { Genome: 120Mb } \\
\text { Pacbio: 11Gb } \\
\text { Illumina: 13Gb }\end{array}$} & CoLoRMap + Miniasm & 963 & 404022 & 60.225 & 0.01853 \\
\hline & FMLRC + Miniasm & 1923 & 57751 & 67.275 & 0.00401 \\
\hline & Jabba + Miniasm & 1632 & 57307 & 62.796 & 0.00041 \\
\hline & LoRDEC + Miniasm & 2232 & 30229 & 43.088 & 0.01107 \\
\hline & LoRMA + Miniasm & 34 & 26316 & 0.361 & 0.00448 \\
\hline & Sprai + Miniasm & 1475 & 169744 & 91.070 & 0.00824 \\
\hline & Miniasm & 740 & 615512 & 0.003 & 0.03409 \\
\hline & Canu & 419 & 835253 & 96.123 & 0.00760 \\
\hline & DBG2OLC & 440 & 754404 & 87.477 & 0.00388 \\
\hline
\end{tabular}

Miniasm does not perform either read correction or consensus calling, so the resulting assembly has the same error profile of the input read

To overcome these limitations, we developed FMLRC, a long read correction method that uses a multi-string BWT and FM-index to represent all de Bruijn graphs of a short read dataset. The method uses two passes to perform the correction: one with a relatively short $k$-mer and one with a longer $K$-mer. In each pass, unsupported sequences are identified in the long reads and the implicit de Bruijn graph identifies alternate, supported sequences from the short reads. These alternate sequences are then used to correct the original read.

We showed that FMLRC reliably corrects more loci than other methods while maintaining competitive gains, sensitivity, and specificity. Furthermore, FMLRC is more computationally efficient than any of the other hybrid 
error-correction methods evaluated. We further showed that using FMLRC as a preassembly error correction step in conjunction with existing overlap-layout assembly methods produces highly contiguous assemblies with competitive accuracy relative to existing hybrid and nonhybrid assembly methods.

Future work will include a specific cost-benefit analysis of the quantity of long- and short-read data required to effectively assemble genomes based on their size and repetitive structure. While previous work has been done in this area, FMLRC, as a more efficient method for hybrid correction of long reads, is expected to allow more effective de novo assembly with less long read data than previously possible. Future improvement and optimization of the FM-Index structure and bridging strategy could produce further speed and accuracy improvements over existing methods. In addition to a BWT with FMindex, it will be worth exploring the performance of other data structures, including novel variants of a de Bruijn graph that support multiple values of $k[27,28]$. Our method is applicable to both Pacbio SMRT sequencing and nanopore sequencing datasets, however further parameter optimization may improve its accuracy and efficiency for nanopore sequences, which exhibit a slightly different error profile than Pacbio. In the long term, better integration of FMLRC error correction along with other tools for overlapping, layout, and consensus of long read sequencing data will help realize the goal of a fully modular and efficient de novo assembly process.

\section{Endnotes}

$$
\begin{aligned}
& { }^{1} \text { http://github.com/holtjma/fmlrc } \\
& { }^{2} \text { http://github.com/holtjma/msbwt } \\
& { }^{3} \text { https://github.com/holtjma/msbwt/wiki/Converting- } \\
& \text { to-msbwt's-RLE-format }
\end{aligned}
$$

\section{Abbreviations}

BWT: Burrows-wheeler transform; ECET: Error correction evaluation toolkit; FMLRC: FM-index long read corrector, Pacbio: Pacific biosciences

\section{Acknowledgements \\ Computational resources were supported by UNC Research Computing (Killdevil and Longleaf clusters).}

\section{Funding}

This work was supported in part by funding from the National Science Foundation (C.D.J., DEB-1457707), North Carolina Biotechnology Center (C.D.J., 2013-MRG-1110), University Cancer Research Fund (C.D.J.), Center for Genome Dynamics (L. M., NIGMS P50 GM076468), and Gastroenterology Basic Science Research Training Program (J.R.W., NIH/NIDDK T32DK007737-17S1).

\section{Availability of data and materials}

We tested the correction algorithms on three publicly available Pacbio datasets. The Pacbio datasets were downloaded for E. coli K12 MG1655, S. cerevisiae W303, and A. thaliana Ler-0 from (https://github.com/ PacificBiosciences/DevNet/wiki/Datasets). For each dataset, we also downloaded complementary short-read sequencing datasets publicly available at: http://spades.bioinf.spbau.ru/spades_test_datasets/ecoli_qmc/ for E. coli, https://www.ncbi.nlm.nih.gov/sra?term=SRR1652473 for A. thaliana, and http://schatzlab.cshl.edu/data/ectools/ for S. cerevisiae.

\section{Authors' contributions}

JRW and CDJ conceived the study. JRW and JH designed the method, performed analyses, and wrote the manuscript. $\mathrm{JH}$ implemented the software. All authors read and approved the final manuscript.

\section{Ethics approval and consent to participate}

Not applicable.

\section{Consent for publication}

Not applicable.

\section{Competing interests}

All authors declare that they have no competing interests.

\section{Publisher's Note}

Springer Nature remains neutral with regard to jurisdictional claims in published maps and institutional affiliations.

\section{Author details}

'Department of Genetics, University of North Carolina at Chapel Hill, CB 3280, 3144 Genome Sciences Building, 250 Bell Tower Dr, Chapel Hill, 27599, NC, USA. ${ }^{2}$ Department of Computer Science, University of North Carolina at Chapel Hill, Chapel Hill, NC, USA. ${ }^{3}$ Department of Biology and Integrative Program for Biological and Genome Sciences, University of North Carolina at Chapel Hill, Chapel Hill, NC, USA.

Received: 8 February 2017 Accepted: 1 February 2018

Published online: 09 February 2018

\section{References}

1. Chin CS, Alexander DH, Marks P, Klammer AA, Drake J, Heiner C, Clum A, Copeland A, Huddleston J, Eichler EE, et al. Nonhybrid, finished microbial genome assemblies from long-read smrt sequencing data. Nature methods. 2013;10(6):563-9.

2. Au KF, Underwood JG, Lee L, Wong WH. Improving pacbio long read accuracy by short read alignment. PLoS ONE. 2012;7(10):46679.

3. Koren S, Schatz MC, Walenz BP, Martin J, Howard JT, Ganapathy G, Wang Z, Rasko DA, McCombie WR, Jarvis ED, et al. Hybrid error correction and de novo assembly of single-molecule sequencing reads. Nat Biotechnol. 2012;30(7):693-700.

4. Salmela L, Rivals E. Lordec: accurate and efficient long read error correction. Bioinformatics. 2014;30(24):3506-14.

5. Berlin K, Koren S, Chin CS, Drake JP, Landolin JM, Phillippy AM. Assembling large genomes with single-molecule sequencing and locality-sensitive hashing. Nat Biotech. 2015;33(6):623-30.

6. Li H. Minimap and miniasm: fast mapping and de novo assembly for noisy long sequences. Bioinformatics. 2016;32(14):2103-110.

7. Miyamoto M, Motooka D, Gotoh K, Imai T, Yoshitake K, Goto N, lida T, Yasunaga T, Horii T, Arakawa K, Kasahara M, Nakamura S. Performance comparison of second- and third-generation sequencers using a bacterial genome with two chromosomes. BMC Genomics. 2014;15(1):1-9.

8. Salmela L, Walve R, Rivals E, Ukkonen E. Accurate selfcorrection of errors in long reads using de bruijn graphs. Bioinformatics. 2016;33(6):799-806.

9. Lee H, Gurtowski J, Yoo S, Marcus S, McCombie WR, Schatz M. Error correction and assembly complexity of single molecule sequencing reads. BioRxiv. 2014006395. https://doi.org/10.1101/006395.

10. Miclotte G, Heydari M, Demeester P, Rombauts S, Van de Peer $Y$, Audenaert $P$, Fostier J. Jabba: hybrid error correction for long sequencing reads. Algo Mole Biol. 2016;11(1):10.

11. Haghshenas E, Hach F, Sahinalp SC, Chauve C. Colormap: Correcting long reads by mapping short reads. Bioinformatics. 2016;32(17):545-51.

12. Goodwin S, Gurtowski J, Ethe-Sayers S, Deshpande P, Schatz MC, McCombie WR. Oxford nanopore sequencing, hybrid error correction, and de novo assembly of a eukaryotic genome. Genome Res. 2015;25(11): 1750-6.

13. Deshpande V, Fung ED, Pham S, Bafna V. Cerulean: A hybrid assembly using high throughput short and long reads. In: International Workshop on Algorithms in Bioinformatics. Springer; 2013. p. 349-63. arXiv preprint arXiv:1307.7933.

14. Ye C, Hill CM, Wu S, Ruan J, Ma ZS. DBG2OLC: efficient assembly of large genomes using long erroneous reads of the third generation sequencing 
technologies. Scientific Reports. 2016;6:31900. https://doi.org/10.1038/ srep31900.

15. Antipov D, Korobeynikov A, McLean JS, Pevzner PA. hybridspades: an algorithm for hybrid assembly of short and long reads. Bioinformatics. 2016;32(7):1009-15.

16. Bauer MJ, Cox AJ, Rosone G. Lightweight BWT construction for very large string collections. In: Giancarlo R, Manzini G, editors. Combinatorial Pattern Matching. CPM 2011. Lecture Notes in Computer Science, vol. 6661. Berlin: Springer; 2011. p. 219-31.

17. Bruijn d. N. A combinatorial problem. Proceedings of the Koninklijke Nederlandse Akademie van Wetenschappen. Series A. 1946;49(7):758.

18. Ferragina P, Manzini G. An experimental study of an opportunistic index. In: Proceedings of the twelfth annual ACM-SIAM symposium on discrete algorithms (SODA '01). Philadelphia: Society for Industrial and Applied Mathematics; 2001. p. 269-78.

19. Burrows M, Wheeler DJ. A block-sorting lossless data compression algorithm. Digital Systems Research Center Research Reports. 1994.

20. Greenstein S, Holt J, McMillan L. Short read error correction using an FM-index. In: 2015 IEEE International Conference on Bioinformatics and Biomedicine (BIBM). Washington: IEEE; 2015. p. 101-4. https://doi.org/10. 1109/BIBM.2015.7359662.

21. Li H. Fast construction of fm-index for long sequence reads. Bioinformatics. 2014;541.

22. Chaisson MJ, Tesler G. Mapping single molecule sequencing reads using basic local alignment with successive refinement (blasr): application and theory. BMC Bioinformatics. 2012;13(1):238.

23. Yang $X$, Chockalingam SP, Aluru S. A survey of error-correction methods for next-generation sequencing. Brief Bioinform. 2013;14(1):56-66.

24. Gurevich A, Saveliev V, Vyahhi N, Tesler G. Quast: quality assessment tool for genome assemblies. Bioinformatics. 2013;29(8):1072-75.

25. Loman NJ, Quick J, Simpson JT. A complete bacterial genome assembled de novo using only nanopore sequencing data. Nat Meth 2015;12(8):733-5.

26. Treangen TJ, Salzberg SL. Repetitive dna and next-generation sequencing: computational challenges and solutions. Nature Rev Gen. 2011;13(1):36-46.

27. Boucher C, Bowe A, Gagie T, Puglisi SJ, Sadakane K. Variable-order de bruijn graphs. In: Proceedings of the 2015 Data Compression Conference, DCC '15. Washington: IEEE Computer Society; 2015. p. 383-92.

28. Cazaux B, Lecroq T, Rivals E. In: Kulikov AS, Kuznetsov SO, Pevzner P, editors. From Indexing Data Structures to de Bruijn Graphs. Cham: Springer; 2014, pp. 89-99.

\section{Submit your next manuscript to BioMed Central and we will help you at every step:}

- We accept pre-submission inquiries

- Our selector tool helps you to find the most relevant journal

- We provide round the clock customer support

- Convenient online submission

- Thorough peer review

- Inclusion in PubMed and all major indexing services

- Maximum visibility for your research

Submit your manuscript at www.biomedcentral.com/submit 